\title{
La modernité de Paul Claudel et la Chine
}

\author{
Zhen Ji \\ Our Lady of the Lake University, San Antonio (Texas)
}

De Claudel à Sollers, en passant par Michaux et Malraux, plusieurs écrivains français du $\mathrm{XX}^{\mathrm{e}}$ siècle essayèrent de trouver en Chine un prétexte ou un support pour mettre en cause l'ancien système occidental et en établir un nouveau. Paul Claudel en fut le premier et probablement un des plus originaux.

Son discours chinois n'a rien de subversif ou militant qu'on trouve chez un Malraux ou chez un Sollers. Par rapport à ses quinze années de séjour en Chine, ses écrits sur la Chine apparaissent peu nombreux, et peu systematiques. En fait une grande partie de ses œuvres chinois sont des rapports ou des discours de conférence. Comme le montrent Jacques Petit et Charles Galpérine dans l'introduction de ses Euvres en prose, Claudel se garde d'exprimer ses idées. («Introduction» xxiv) Même s'il lui faut se faire une opinion, il préfère des suggestions aux thèses définitives. Toutefois cette pensée à " l'état naissant " révèle des idées très avancées sur la culture chinoise et sa relation avec sa contrepartie occidentale. Ses « rêveries ", ses suggestions ou ses propositions à l'égard du langage et de la notion de l'homme sont d'autant plus importantes qu'elles émanent d'une sensibilité très moderniste. Entre une littérature d'" inventaires et de descriptions " d'antan et une nouvelle forme d'" énonciation scénique " et d'« équation typographique $^{1}$ » Paul Claudel n'hésite pas à suivre les pas de son vieux maître, en reprenant ce refrain : "Imiter le Chinois au cœur limpide et $\operatorname{fin}^{2} »$.

Parlons d'abord de ses points de vue sur le langage. Claudel est le premier écrivain français qui jette un regard tout nouveau sur l'écriture chinoise en le prenant comme un système. Avant lui Voltaire n'y a vu que difficulté et obstacle pour la communication et l'échange. Déjà dans La religion du signe, il aperçoit ce qui est 


\section{Zhen Ji}

différent entre les deux écritures. Pour lui le mot occidental est analytique, et à l'opposé, le signe chinois est visuel. L'un se développe dans la succession, l'autre dans la proportion de ses traits, c'est-à-dire dans la dimension spatiale. Pour lui, le signe chinois jouit aussi d'une indépendance que l'écriture occidentale ne possède pas. C'est une totalité : « le Caractère Chinois est un être schématique ». Le titre même de ce poème met l'accent sur le statut primordial, sacré de l'écriture chinoise. Henri Michaux écrira dans le même sens : "Chine, pays où l'on méditait sur les tracés d'un calligraphe, comme en un autre pays on méditera sur un mantra, sur la substance, le principe, ou sur l'Essence. " ( « Idéogrammes en Chine » 109)

Il reprend son éloge de l'écriture chinoise dans « La philosophie du livre » en indiquant : «Ce n'est pas sur les papiers de l'Occident que le mot, la macule intelligible sur le blanc arrive à sa pleine gloire, à sa signification rayonnante et stable. Il n'est qu'une potion mal apaisé de la phrase, un tronçon du chemin vers le sens, un vestige de l'idée qui passe. Il nous invite à ne pas nous arrêter nous-mêmes, à continuer jusqu'au point final le mouvement des yeux et de la pensée. Le mot chinois, au contraire, cette image abstraite de la chose, cette clef de la détermination et de l'idée, reste aussi fixe devant l'oil du contemplateur que le pentacle lumineux qu'envisage le Docteur Faustus dans l'estampe de Rembrandt ». ( "La philosophie du livre » 75) En d'autres termes, l'écriture occidentale est supplémentaire et insignifiante, étant le porteur d'un sens, d'une « idée qui passe »; tandis que l'écriture chinoise retient plus l'attention du lecteur, grâce à sa stabilité et sa visibilité picturale.

Ce qui est remarquable, c'est que tout en applaudissant le rayonnement du signe chinois, il voit dans la langue européenne la possibilité d'un langage poétique. Le livre du P. Wieger semble réveiller chez notre poète cette fierté pour la langue française, qui est, selon lui, aussi mimétique que le chinois. "J'ai été amené à me demander si dans notre écriture occidentale il n'y aurait pas moyen de retrouver également une certaine représentation des objets qu'elle signifie » («Idéogrammes occidentaux » 81), [...] « et tout 
à coup une idée me frappa. Mais nous aussi nous avons des idéogrammes et nos langues sont tout aussi propres que le chinois à donner une représentation graphique des objets. » (« L'harmonie imitative » 101) Selon lui, si le caractère chinois peut se diviser en plusieurs parties signifiantes et qu'on retrouve un sens complet grâce à ce lien créé entre les traits et la réalité, le mot français peut aussi se lire par chaque lettre séparée, laquelle, nouant un lien mimétique avec la chose que désigne le mot, fait de celui-ci une unité pleine comme le caractère chinois. Comparons son interprétation du caractère «水》(shui) et celle du mot «pain »:

Le caractère qui veut dire l'eau en chinois est un gribouis conventionnel représentant le mouvement d'un liquide. Le pinceau du scribe ajoute un point sur le côté, cela veut dire la glace $e^{3}$ Il met le point en haut : cela veut dire toujours, l'éternitét Ainsi ce qui était le mouvement par excellence est solidifié dans une espèce de permanence abstraite, comme la cascade que la distance nous fait paraître immobile. Il n'y a pas de caractère chinois où ce point ne soit sous-entendu. ( La philosophie du livre $\gg 72$ )

$P$ est la pelle qui enfourne ou encore la langue tendue vers la bonne niche, a est la niche même ou la bouche emmanchée sur le cou ou encore le geste circulaire du mitron qui pétrit, $\mathrm{i}$ est le feu ou la faim, $\mathrm{n}$ est la voûte du four ou les dents enfoncées. - et les quatre lettres ne forment qu'une seule syllabe comme de la pâte. (83)

Cet attachement de Claudel au mimétisme va de pair avec sa revendication de la réforme de la poésie française. Pour lui, il faut libérer le vers de la contrainte de la rime, de la césure fixe, du nombre fixe de syllabes. Il est temps d'introduire plus de jeu et de hasard, de s'appuyer davantage sur la « valeur première et incantatoire du langage », sur le pouvoir suggestif des images. On a surtout besoin de donner la primauté à l'expression, au mot, à la phrase, à 


\section{Zhen Ji}

la forme poétique. L'esprit de Mallarmé, note-t-il, va toujours des mots aux choses, non pas des choses aux mots. Le mot a une âme, insiste-t-il. C'est ainsi qu'il évoque le pouvoir d'un mot idéal dans ces vers :

Un mot rond sans aucune tige comme un lotus qui s'épanouit tout seul en plein papier, un seul caractère que le doigt n'achève pas sur le sable,

Et l'âme tout entière s'émeut dans les profondeurs superposées de son intelligence. ( La philosophie du livre » 73)

Ce mot idéal, on le voit en fait s'ouvrir sur les pages de Cent phrases pour éventails, où Claudel pousse plus loin son sentiment d'émulation à l'égard de l'écriture pictographique. Quoique notre auteur ne soit pas le premier poète occidental qui introduit la calligraphie chinoise dans ses poèmes, la présence de ces signes calligraphiés semble servir à un but plus précis que chez ses devanciers ${ }^{5}$. Ils sont là pour rappeler cet effet de simultanéité propre à l'écriture chinoise. Notre poète s'explique : le mot occidental est « un ensemble obtenu par une succession », il crée un parcours dans le temps, il va directement au lecteur; tandis que le caractère chinois propose une "dilatation dans le blanc ", il évoque, autour de lui, une constellation graphique. Le rapport entre les mots n'est pas analytique, mais visuel. Cependant il est tout à fait possible de créer cet effet de simultanéité du signe pictographique. Il suffit d'introduire cette deuxième dimension dans l'écriture. Il propose :

Laissons à chaque mot, qu'il soit fait de seul ou de plusieurs vocables, à chaque proposition verbale, l'espace- le tempsnécessaire à sa pleine sonorité, à sa dilatation dans le blanc. Que chaque groupe ou individu graphique prenne librement sur l'aire attribuée l'habile position qui lui convient par rapport aux autres groupes? Substituons à la ligne uniforme un libre ébat au sein de la deuxième dimension! Et puisque c'est la pensée seule par une espèce de choc en retour qui solidifie les successifs éléments du 
mot, pourquoi ne pas retarder quand il le faut par un espacement calculé la résolution du noir caillot intellectuel et prolonger l'insistance de l'appel qu'il articule? (Cent phrases pour éventails 701)

Notons que de Mallarmé au groupe Tel Quel, l'effort de toute cette littérature avant-gardiste n'est rien d'autre que de substituer la linéarité des vers, des phrases par « un libre ébat au sein de la deuxième dimension ", c'est-à-dire couper le souffle et la voix, faire émerger l'écriture textuelle.

Du blanc de la page, passons à une autre notion du vide, c'est la vacuité intérieure de l'homme. Pour Claudel, l'homme est vide sans Dieu. Celui-ci est la seule chose solide sur laquelle l'homme peut compter. En adorant Dieu, l'homme trouverait tout, la joie, la paix, l'ordre... Rien ne peut remplir le vide qui est laissé par l'absence de Dieu. Ni Justice, ni Progrès, ni Vérité, ni l'Humanité, ni Lois de la Nature, ni Art, ni Beauté. Le bouddhisme n'ira pas non plus, car Dieu y est absent. C'est pourquoi il existe dans Connaissance de l'Est ce double visage de l'homme. D'un côté, il y a l'image de cette créature fragile qui, à tout moment, risque d'être engloutie par l'Abîme, emporté par les « élations de la profondeur et du Vent "; de l'autre, le profil de ce poète jubilant, qui vit en connivence avec la terre chinoise, et se fait le chantre de Dieu et l'harmonie de ce monde.

Cependant, aux yeux de Claudel, l'homme a un autre vide à remplir, à part le vide laissé par Dieu. Ainsi écrit-il dans «Théorie de co-naissance »:

L'être animé est creux; à la façon d'une bouteille, il témoigne du souffle qui a formé et le regonfle à chacune de ses aspirations. Ce vide comporte un état de déséquilibre natif, une démolition interne et passive, compensée par une reprise active sur le dehors. (Art poétique 162)

Croyant que l'homme est un être sans fond, Claudel trouve la maxime socratique « connais-toi toi-même " absurde. "On ne se connaît pas soi-même, affirme-t-il, parce que le fond de soimême n'est rien, c'est le néant. Le vrai moyen de connaissance 
serait plutôt : "Oublie-toi toi-même", "oublie-toi toi-même pour être absorbé dans le spectacle qui s'offre à toi et qui est infiniment plus intéressant [...]" » (Mémoires improvisés 198) À ses yeux c'est dans la perception du monde extérieur que l'homme a la chance de se connaître ou plus précisément de prendre conscience de sa place dans le monde. Car dans la perception des choses, c'est tout l'être qui fonctionne, et ses sens, son intelligence, son imagination qui entrent dans un mouvement continuel de vibration. C'est dans cet état d'oscillation que l'être ne cesse de co-naître, et de naître. Ainsi s'explique Claudel :

\begin{abstract}
Toute connaissance est une : " naissance », par une espèce de jeu de mots qui, somme toute, est confirmé par l'étymologie : " connaissance » est un « co-naissance ». Nous ne cessons pas de co-naître au monde, c'est-à-dire que notre connaissance c'est l'œuvre de l'épanouissement circulaire de notre être constamment en état de vibration, sur lequel viennent s'insérer les touches diverses qui sont les objets de cette co-naissance spéciale. (Art poétique 195)
\end{abstract}

De cette co-naissance au monde, l'homme pourrait se faire une idée de sa place au monde, et aussi de sa valeur en tant que « point de coordination des phénomènes divers auxquels il apporte des témoignages communs ». (Art poétique 170) Dans cette perspective, Connaissance de l'Est devrait se comprendre comme un chant de la co-connaissance du poète en pays de l'Est, qui se sent à tout instant naître et renaître au contact de son entourage, dans la prise de conscience de l'existence des banyans, des pins, des fleuves qui coulent, et des paysans qui travaillent aux champs.

Il n'est pas sûr que la notion claudélienne du vide intérieur de l'homme est en rapport direct avec la notion du vide du taoïsme. Ce qui est certain, c'est qu'inité par le Daodejing, notre écrivain apprend très vite à voir le vide dans son aspect fonctionnel et constitutionnel, et à le saisir dans les nuances les plus subtiles. Comme le montre ce passage qu'il écrit au début de son séjour en Chine : 
La Chine montre partout l'image du vide constitutionnel dont elle entretient l'économie. "Honorons ", dit le Tao teh king, « la vacuité qui confère à la roue son usage, au luth son harmonie. » Ces décombres et ces jachères que l'on trouve dans une même enceinte juxtaposés aux multitudes les plus denses, à côté de minutieuses cultures ces monts stériles et l'étendue infinie des cimetières, n'insinuent pas dans l'esprit une idée vaine. Car dans l'épaisseur et la masse de ce peuple cohérent, l'administration, la justice, le culte, la monarchie, ne découvrent pas par des contrastes moins étranges une moins béante lacune, de vains simulacres et leurs ruines. (Connaissance de l'Est, 78)

Il essaie aussi de confondre les notions du vide, du tao et de l'âme, trouvant sans doute dans le mélange la possibilité d'introduire une portée religieuse. Par exemple dans cette présentation du vase chinois :

L'essence du vase chinois est d'être vide [...]

L'importance du vide, c'est toute la philosophie, c'est tout l'art chinois. Le vide en tout être, c'est le chemin mystique, c'est le tao, c'est l'âme, c'est la tendance, c'est l'aspiration mesurée, à quoi le vase donne sa forme la plus parfaite, réalisant la thèse d'A ristote que l'âme est la forme du corps. Considérons-le dans son gabarit le plus typique. Le vase comporte trois parties : le récipient ou panse, le col le plus ou moins allongé, qui représente l'aspiration, et la corolle plus ou moins épanouie qui est l'expansion vers l'invisible, le débouché ou, si vous aimez mieux, l'abouchement avec l'esprit. («AEGRI SOMNIA » 892-893)

En insistant à ce que l'homme ne se connaît que par les autres, Claudel fait une rupture avec le cogito cartésien. Si pour Descartes 
la devise était « Je pense, donc je suis ", pour Claudel ce serait plutôt « Je suis ce que je ne suis pas » ou « Être, c'est connaître ». À l'homme indépendant de Descartes, Claudel oppose l'homme interdépendant. Son abandon du cogito cartésien l'entraîne aussi à rejeter le syllogisme et à adopter une nouvelle logique. Claudel la précise dans le passage suivant :

\begin{abstract}
Jadis au Japon, comme je montais de Nikkô à Chazenji, je vis, quoique grandement distants, juxtaposés par l'alignement de mon œeil, la verdure d'un érable combler l'accord proposé par un pin. Les présentes pages commentent ce texte forestier, l'énonciation arborescente, par Juin, d'un nouvel Art Poétique de l'Univers, d'une nouvelle Logique. L'ancienne avait le syllogisme pour organe, celle-ci (la nouvelle) est la métaphore, le mot nouveau, l'opération qui résulte de la seule existence conjointe et simultanée de deux choses différentes. (Art poétique 143)
\end{abstract}

Dans Claudel et l'univers chinois Gilbert Gadoffre suggère que Saint Bonaventure, Huysmans et Mallarmé aient inspiré Claudel dans son adoption de cette analogie. Il est possible que Claudel ait subi également une influence chinoise. Car cette nouvelle logique présente une parenté surprenante avec celle de la cosmologie chinoise. Son discours intitulé "Les superstitions chinoises"le montre bien informé du fond de cette pensée chinoise. Il comprend que l'essence de la philosophie chinoise repose sur la conception de ces deux principes opposés, le yin et le yang, et qu'aux yeux des Chinois, leur transformation éternelle constitue l'évolution de l'univers. Il sait également que le yin et le yang sont capables de représenter les deux aspects contraires d'une même chose, et que leur rapport est aussi contigu et complémentaire : «Au moment où le yang est à son apogée [...] l'autre se substitue à lui insensiblement [...] Chacune porte en soi le germe de l'autre $[\ldots] »$ (« Les superstitions chinoises »1081) 
Ce glissement de Claudel de la métaphysique occidentale à la cosmologie chinoise est sans aucun doute très révolutionnaire, si l'on pense à tout ce tapage fait par le groupe de Tel quel et les poststructuralistes au sujet de la Chine dans les années 60 et 70 du siècle dernier. La pensée chinoise est utilisée par eux comme une arme contre la métaphysique occidentale et la tradition humaniste. Mais Claudel n'est pas aussi radical qu'eux. Il se contente d'une sorte d'éclectisme, parlant de la gloire de Dieu tout en confondant le vide, le tao et la notion d'âme. C'est le même avec son expérimentation d'écriture poétique, qui reste un jeu, un « amusement d'un jour de pluie ". Il ne va pas aussi loin qu'un Sollers, pas même qu'un Mallarmé. Car il comprend que par-delà l'abstention, il n'y a que l'absence ${ }^{6}$ ( «Mallarmé » 511)

\section{Notes}

1 Voir Paul Claudel, "Mallarmé » in CEuvres en prose, p. 511, Gallimard, 1965.

2 «Imiter le Chinois au cœur limpide et fin

De qui l'extase pure est de peindre la fin

Sur ses tasses de neige à la lune ravie

D'une bizarre fleur qui parfume sa vie

Transparente, la fleur qu'il a sentie, enfant,

Au filigrane bleu de l'âme se greffant.

Et, la mort telle avec le seul rêve du sage,

Serein, je vais choisir un jeune paysage

Que je peindrais encor sur les tasses, distrait.

Une ligne d'azur mince et pâle serait

Un lac, parmi le ciel de porcelaine nue,

Un clair croissant perdu par une blanche nue

Trempe sa corne calme en la glace des eaux,

Non loin de trois grands cils d'émeraude, roseaux. "

Cité par Paul Claudel in « Poésie française et Extrême-Orient », Euvres en prose, p. 1042.

3 冰 en caractère chinois.

4 永 en caractère chinois. 


\section{Zhen Ji}

$5 \mathrm{Au}$ moment où Claudel écrit la préface pour la réédition de Cent phrases pour éventails, le monde occidental a vu au moins deux ceuvres poétiques où les signes chinois sont inscrits : Les Stèles de Segalen et Les Cantos d'Ezra Pound.

6 Claudel écrit à propos de Mallarmé : "Sous la copieuse machine des apparences il y a en réalité vacance, absence. [...] Et sans doute que le meilleur moyen d'exprimer l'absence, c'est encore l'abstention. " Euwres en prose, p. 512.

\section{Bibliographie}

Claudel, Paul. «Religion du signe ». Euvre poétique. Paris : Gallimard, 1967.

—. Connaissance de l'Est, CEuvre poétique. Paris : Gallimard, 1967.

—. "Magnificat ". Cinq grandes Odes. Euvre poétique. Paris : Gallimard, 1967.

—. Cent phrases pour éventails. Euvre poétique. Paris : Gallimard, 1967.

—. Art poétique. Euvre poétique. Paris : Gallimard, 1967.

—. «ur l'inspiration poétique ». Euure en prose. Paris : Gallimard, Collection « La Bibliothèque de la Pléiade », 1965.

-. «La philosophie du livre». Euvre en prose. Paris : Gallimard, Collection « La Bibliothèque de la Pléiade », 1965.

—. "Les mots ont une âme ». (Euvre en prose. Paris : Gallimard, Collection «La Bibliothèque de la Pléiade », 1965.

—. «L'harmonie imitative». Euvre en prose. Paris : Gallimard, Collection « La Bibliothèque de la Pléiade », 1965.

—. Paul Claudel, « Idéogrammes occidentaux » Euvre en prose. Paris : Gallimard, Collection « La Bibliothèque de la Pléiade », 1965.

—. «AEGRI SOMNIA ». Conversation. Euvre en prose. Paris : Gallimard, Collection « La Bibliothèque de la Pléiade », 1965.

—. «Le poète et le vase d'encens". Conversation. CEuvre en prose. Cuvre en prose. Paris : Gallimard, Collection « La Bibliothèque de la Pléiade », 1965.

——. "La poésie française et l'Extrême-Orient ». Euure en prose. Paris : Gallimard, Collection « La Bibliothèque de la Pléiade », 1965.

- «Les superstitions chinoises ». Contacts et circonstances. Euvres en prose. Paris : Gallimard, 1965. 


\section{La modernité de Paul Claudel et la Chine}

—. Mémoires improvisés. Recueillis par Jean Amrouche, Paris : Gallimard, 1954.

Derrida, Jacques. De la Grammatologie. Paris : Minuit, 1967.

«Sémiologie et grammatologie ». Position. Paris : Minuit, 1972.

Domenach, J.-M. Le Tombeau de l'humanisme. Paris : Seuil, 1976.

Ferry, Luc et Alain Renault. La pensée 68. Paris : Gallimard, 1985.

Gadoffre, Gilbert. Claudel et l'univers chinois, Cahiers Paul Claudel. Paris :

Gallimard, 1968.

Goux, Jean-Joseph. Les monnayeurs du langage. Paris : Galilée, 1984.

Lao Tse. Tao te king ou Livre de la Voie et de la Vertu. Traduction de Stanislas Julien. Paris : Éditions Mille et une nuits, 1996.

Malraux, André. La tentation de l'Occident. Paris : Grasset, 1964.

Michaux, Henri. Un barbare en Asie. Paris : Gallimard, 1945.

Sollers, Philippe. "Pourquoi j'ai été Chinois ». Improvisations. Paris : Gallimard, 1991.

—. «L'évidence chinoise ". L'infini. N. 79 Été 2002, Gallimard.

Wieger, Léon. Les textes historiques. Hien-Hien, 1903.

-. Les Caractères chinois : étymologie, graphie, lexiques. Taichung : Kuangchi Press, 1963. 